\title{
Technik zur Unterstützung von Citizen Science und Open Science
}

\author{
Technische und organisatorische Herausforderungen \\ und mögliche Lösungsansätze
}

\author{
Karsten Weber, Institut für Sozialforschung und Technikfolgenabschätzung, Ostbayerische Technische Hochschule Regensburg, \\ Galgenbergstraße 24, 93053 Regensburg (Karsten.Weber@oth-regensburg.de), (1) orcid.org/0000-0001-8875-2386 \\ Nadine Kleine, Institut für Sozialforschung und Technikfolgenabschätzung, Ostbayerische Technische Hochschule Regensburg \\ (Nadine.Kleine@oth-regensburg.de), (1) orcid.org/0000-0003-1761-4810 \\ Frank Pallas, Institut für Wirtschaftsinformatik und Quantitative Methoden - Information Systems Engineering, \\ Technische Universität Berlin (Frank.Pallas@tu-berlin.de), (1) orcid.org/0000-0002-5543-0265 \\ Max-R.Ulbricht, Institut für Wirtschaftsinformatik und Quantitative Methoden - Information Systems Engineering, \\ Technische Universität Berlin (mu@ise.tu-berlin.de), @ orcid.org/0000-0001-7134-4351
}

Wenn BürgerInnen aktiv am Datengewinnungsprozess als zentralem Baustein empirisch ausgerichteter wissenschaftlicher Projekte teilhaben, kann dies als Beitrag zu einer offenen und bürgernahen Wissenschaft angesehen werden. Eine solche Teilhabe kann durch die Bereitstellung von technischen Werkzeugen erheblich erleichtert werden. Daher sollen Participatory Sensing als Bereitstellung von günstigen Sensoren zur Messung von Umweltparametern sowie Wearable Technologies zur Aufnahme von quantifizierten Vitaldaten und physiologischen Zuständen vorgestellt werden. Konzeptionell kann die Bereitstellung von Daten, die mit diesen Werkzeugen erhoben wurden, als Allmende verstanden werden - mit allen damit verbundenen Chancen und Risiken. Nach der Beschreibung von Beispielen aus den Bereichen von Participatory Sensing und Wearable Technologies werden zu erwartende Herausforderungen identifiziert und technisch-organisatorische Ansätze zu deren Lösung skizziert.

Participatory sensing and wearable technologies as tools to support citizen and open science

Technical and organizational challenges and possible solutions

If citizens actively participate in the process of collecting empirical data, as a key element of empirically oriented scientific projects, this can be seen as a contribution to an open and citizen-oriented science. Such participation can be supported by providing technical tools. The paper therefore presents examples of participatory sensing as the provision of affordable sensors for measuring environmental parameters as well as wearable technologies for recording quantified vital data and phys-

This is an article distributed under the terms of the Creative Commons Attribution License CCBY 4.0 (https://creativecommons.org/licenses/by/4.0/)

https://doi.org/10.14512/tatup.26.1-2.25

Eingereicht: 13. 03.2017. Angenommen: 15. 05.2017 iological states. Conceptually, the provision of data collected with these tools can be understood as a commons - with all opportunities and risks associated with such goods. After describing examples of participatory sensing and wearable technologies, the authors identify potential challenges and outline technical and organizational approaches to solve them.

KEYWORDS: participatory sensing, wearable technology, citizen science, open science

\section{Einleitung}

Participatory Sensing steht für die Einbeziehung von BürgerInnen bei der Erfassung und Dokumentation von Umgebungsbedingungen. Als aktuelle Beispiele können die Initiativen zur Messung von Radioaktivität rund um das belgische AKW Tihange - Tihange Doel Radiation Monitoring (tdrm.fiff.de) auf belgischer und die Bürgerinitiative MAUS, Messen für aktiven Umweltschutz (www.maus-trier.de), auf deutscher Seite - genannt werden. Solche Initiativen können dazu beitragen, Umweltveränderungen $\mathrm{zu}$ dokumentieren und Wirkungszusammenhänge aufzudecken, um so Bürgerinteressen gegenüber politischen Entscheidungsträgern besser einfordern zu können. Wearable Technologies in Form von kleinen, direkt am Körper getragenen Sensoren, die beispielsweise Vitaldaten des Trägers und Informationen aus der direkten Umgebung erfassen, ermöglichen es wiederum, physiologische Zustände und Aktivitäten quantifiziert aufzunehmen und auszuwerten. Im Gesundheitswesen ergeben sich hieraus neue Möglichkeiten zur Prävention, 
Erkennung und Behandlung, da ein dauerhaftes und ständiges Monitoring verspricht, Krankheitssymptome frühzeitig erkennen und gegebenenfalls gezielter intervenieren zu können. So propagiert die bereits 2007 begründete und weltweit aktive Quantified Self-Bewegung (www.quantifiedself.com) die Nutzung von Wearables und anderen Technologien zur Selbstvermessung und den Datenaustausch mit dem Slogan „self knowledge through numbers".

In beiden Fällen spielt die Teilhabe der BürgerInnen am Datengewinnungsprozess als zentralem Baustein empirischer Wissenschaft eine herausgehobene Rolle. Participatory Sensing und Wearable Technologies können somit einen Beitrag zu einer offenen Wissenschaft (Open Science) mit starker Bürgerbeteiligung (Citizen Science) leisten. Inwiefern dies von derzeit diskutierten Ansätzen bspw. zur Datenbereitstellung und -auswertung oder der Rekrutierung von Beteiligten im Kontext von Participatory Sensing und Wearable Technologies tatsächlich unterstützt wird, soll auf den nächsten Seiten untersucht werden.

Im Folgenden wird hier nicht ausführlich auf Open Science und Citizen Science sowie die relevanten Akteure eingegangen dies wird in anderen Beiträgen für die vorliegende TATuP-Ausgabe geleistet (Riehm und Nentwich; Häußermann und Heidingsfelder; Vohland und Göbel in diesem Heft). Nur so viel: Es wird davon ausgegangen, dass Citizen Science stets auch Open Science umfasst (zur Definition siehe Fecher und Friesike 2014) - umgekehrt gilt dies jedoch nicht notwendigerweise. Als wesentliche Eigenschaft von Citizen Science wird meist genannt, dass nicht nur professionelle WissenschaftlerInnen Forschung betreiben, sondern BürgerInnen in den Erkenntnisprozess mit einbezogen werden. Vayena und Tasioulas (2015, S. 480) führen dabei zahlreiche Anwendungsbereiche auf, ebenso das Grünbuch Citizen Science Strategie 2020 für Deutschland (GEWISS 2016), die im weitesten Sinne im Bereich des Umweltschutzes oder der Umweltbeobachtung liegen. Ein recht bekanntes Beispiel ist das Zählen von Vögeln, das jährlich vom Naturschutzbund Deutschland organisiert wird (NABU 2016). An diesem und vielen anderen Projekten lassen sich Merkmale von Citizen Science aufzeigen, die für die folgenden Überlegungen wichtig sein werden:

1. Es sollen viele BürgerInnen eingebunden werden, damit umfangreiche Datensätze erhoben werden können.

2. Sofern es sich nicht nur um ein lokales Phänomen handelt, sollen diese BürgerInnen weiträumig verteilt sein, damit das Phänomen in Gänze beobachtet werden kann.

3. Daten sollen möglichst einfach erhoben werden können, so dass niemand mangels fehlender Ressourcen ausgeschlossen wird.

4. Alle erhobenen Daten sollen wiederum für alle BürgerInnen frei zur Verfügung stehen.

Es gibt sicherlich weitere Eigenschaften bzw. Ziele von Open und Citizen Science, die aus Perspektive der Science and Technology Studies oder der Innovationsforschung von Bedeutung sein mögen (Lindeman 2012; Ruiz-Mallén et al. 2016; Scott 2015) - im Folgenden werden jedoch diese vier genannten Merkmale genauer betrachtet, da deren Erreichung durch Einsatz und konkrete Ausgestaltung einer technischen Infrastruktur erleichtert werden kann.

\section{Technik für Citizen Science und Open Science}

Im Folgenden wird primär die Bereitstellung von Sensoren betrachtet; auf darüber hinaus notwendige Infrastrukturen wie Plattformen, gemeinsame Datenformate, Server etc. kann nicht näher eingegangen werden. Viele der weiter unten aufgeführten Herausforderungen stellen sich bei Betrachtung einer Open Science- bzw. Citizen-Science-Infrastruktur in ähnlicher Weise.

\section{Participatory Sensing}

Participatory Sensing ist eng mit der Verfügbarkeit der in modernen Smartphones enthaltenen Sensoren (GPS, Mikrofon, Kamera, Bewegungs- und Beschleunigungssensoren etc.) verbunden (Goldman et al. 2009; Devarakonda et al. 2013). Allerdings sind diese Geräte nicht die einzigen technischen Möglichkeiten zur Umsetzung; die Erfassung von Umgebungsparametern wie Temperatur, Helligkeit, Luftfeuchtigkeit und Geräuschpegel ist bereits für weniger als 150 Euro und ohne übermäßiges technisches Fachwissen durch intelligente Messstationen realisierbar.

Als Projektbeispiele können u. a. die SenseBox vom Institut für Geoinformatik der Universität Münster (www.sensebox. de), das Oxford Flood Network (www.oxfloodnet.co.uk) sowie das AirQualityEgg (www.airqualityegg.com) genannt werden. Sie zielen darauf ab, Daten über Umweltparameter zu erheben und frei zur Verfügung zu stellen. Bei SenseBox stehen Bildung und das Heranführen von SchülerInnen an Sensortechnik im Vordergrund, doch die Technik könnte durchaus als Basis eines weitgefächerten Sensornetzwerkes dienen. Das Oxford Flood Network soll dazu beitragen, genauere Informationen über die Pegelstände der Flüsse Themse und Cherwell zu sammeln, um so langfristig bessere Prognosen über mögliche Überflutungen geben und zeitnah vor diesen warnen zu können (Pacheco-Torgal 2016, S. 422 ff.). Das Projekt AirQualityEgg (Austen 2015; Kumar et al. 2015) soll Luftgüteparameter wie Stickstoffdioxid-, Kohlenstoffmonoxid- oder Feinstaubgehalt liefern, um eine umfängliche Dokumentation der Luftqualität in belasteten Städten zu ermöglichen. In allen Fällen werden die Sensor- und Kommunikationseinheiten üblicherweise von Freiwilligen auf eigene Kosten installiert und betrieben.

\section{Wearable Technologies}

Ausgehend von (Fitness-)EnthusiastInnen, die mit Schrittzählern, Personenwaagen oder Herzfrequenzmessern entsprechend quantifizierbare Aspekte ihres Fitness- und Gesundheitszustandes dokumentieren und so optimieren wollten (Vaz und Bruno 
2003), wurde die Quantified-Self-Bewegung durch das Aufkommen sogenannter Wearable Technologies schnell zu einem weltweiten Phänomen. Tragbare Sensoren, Clips zum Befestigen an der Kleidung, Brustgurte oder Einsätze in Laufschuhen und Shirts ermöglichen zusammen mit Applikationen für Smartphones oder Tablet-PCs den ursprünglich manuellen Vorgang des Datensammelns und -auswertens zu automatisieren. Via Gamification, also der Verbindung des Selbstvermessens mit Elementen des Wettkampfs mit anderen Personen (Whitson 2013), soll die Teilnahmebereitschaft gesteigert werden; die Verbindung mit digitalen sozialen Netzwerken erleichtert dabei den Austausch von Daten. Gemessen, gesammelt und verglichen werden bspw. Bewegungs- und Positionsdaten, Vitaldaten wie Blutdruck und Herzfrequenz, aber auch zurückgelegte Strecken und Schlafverhalten. Auf diese Weise wird ein immer umfassenderes und genaueres Bild der eigenen, nun quantifizierten Lebensumstände geschaffen (Swan 2012). Mithilfe moderner Analyseverfahren lassen sich auf Basis dieser Daten zum einen bisher unbekannte Zusammenhänge bestimmter Einzelaspekte des individuellen Lebens und des Gesundheitszustands aufdecken. Zum anderen ist es aber durch Kombination unterschiedlicher individueller Wearable-Datensätze möglich, neue Arten gesundheitsbezogener Breitenstudien durchzuführen.

\section{Gemeinsamkeiten}

Meist besteht bei den genannten Beispielen zuallererst ein primäres Eigeninteresse der jeweiligen Akteure an der Datenerhebung. Menschen haben ein generelles Interesse z. B. an der Luftqualität ihres Wohnortes oder ihrem Fitnesszustand und investieren daher eigenes Geld in entsprechende Sensorik. Gerade im Bereich des Participatory Sensing, vermehrt aber auch im Fall von Wearable Technologies, besteht darüber hinaus zunehmend auch ein sekundäres Interesse, die erfassten Sensordaten nicht nur für den primären Zweck zu verwenden, sondern als Rohdaten frei zugänglich zur allgemeinen Nutzung bereitzustellen. Hierzu existiert eine Reihe spezialisierter Webplattformen. Solche Plattformen stellen öffentliche Programmierschnittstellen zur Verfügung, die es erleichtern können, bereitgestellte Daten abzurufen, um auf deren Basis gänzlich neue Zusammenhänge herzustellen. Damit sind die zu Beginn genannten Charakteristika im Prinzip erfüllt: Die hier vorgestellten Beispiele zu Wearable Technologies haben, neben der individuellen Nutzung, auch das sekundäre Ziel, dass (1) möglichst viele BürgerInnen durch die Bereitstellung günstiger Technik in den Datenerhebungsprozess eingebunden werden; (2) diese weiträumig verteilt sind, um möglichst viele und repräsentative Daten erheben zu können; (3) Daten durch einfach zu bedienende Technik leicht erhoben werden können; und (4) möglichst viele erhobenen Daten wiederum für möglichst zahlreiche interessierte Personen frei zur Verfügung stehen. Participatory Sensing hingegen zielt primär auf diese vier Punkte, da es stets die Einbeziehung der Bürger bei der Erhebung, Dokumentation, Verarbeitung und Verbreitung von Daten über deren eigene Lebensumstände betont (Goldman et al. 2009, S.4).

\section{Herausforderungen}

Aus der Struktur und Anwendungsweise von Participatory Sensing und Wearable Technologies als Werkzeuge für Citizen Science und Open Science ergeben sich einige technische wie nichttechnische Schwierigkeiten, wegen derer sich Offenheit und Mitwirkungsmöglichkeiten - die eigentlichen Stärken dieser Ansätze - unter bestimmten Umständen in Schwächen wandeln können. Eine klare Trennung technischer wie nichttechnischer Herausforderungen ist nur bedingt möglich, da sich diese meist wechselseitig bedingen. Bewusst wird im Folgenden nicht auf Fragen des Datenschutzes oder praktische Fragen der fehlerhaften Bedienung eingegangen. Stattdessen soll betont werden, dass die Möglichkeit der Kooperation nicht notwendig zum Gelingen der Kooperation führt - auch nicht in technisch unterstützten Fällen.

\section{Die Tragik der digitalen Allmende}

Ähnlich wie Open-Source-Software lassen sich die Sensoren, zumindest aber die von diesen generierten und öffentlich bereitgestellten Daten, als digitale Allmende verstehen. Daher kann die gesamte Theoriebildung zu Allmende- bzw. öffentlichen Gütern (ausgehend von Olson 1965 und Hardin 1968) herangezogen werden. Ein Problem dieser Güter sind fehlende Anreize für Beiträge zu deren Herstellung, so dass viele Menschen sie zwar nutzen, aber nicht zur Produktion beitragen - dieses Trittbrettfahrerproblem ist Teil der sogenannten Tragik der Allmende (Hardin 1968) und führt auf längere Sicht wiederum zur Zerstörung entsprechender Güter. Free riding wird oft durch fehlende Regeln für die Nutzung eines Gutes bzw. durch deren (vermeintliche) Kostenlosigkeit begünstigt. Ein weiteres Problem ist die Unternutzung durch zu starke Regulierung eines Allmende- bzw. öffentlichen Gutes (Buchanan und Yoon 2000).

Angewendet auf Participatory Sensing und Wearable Technologies taucht Trittbrettfahren dann auf, wenn Individuen oder auch Organisationen diese Infrastruktur für eigene, beispielsweise kommerzielle, Zwecke nutzen, aber selbst keinen Beitrag zur Aufrechterhaltung der Technik oder zur Verbesserung der Datenbasis leisten. Dies könnte so weit gehen, dass sich beispielsweise staatliche Institutionen auf die private Bereitstellung von Sensoren und Daten verlassen. Andererseits könnten strikte Regeln, beispielsweise eine Anmeldepflicht für Participatory Sensing und Wearable Technologies, mit dem Ziel der Verhinderung von Trittbrettfahren, dazu führen, dass das Betreiben und die Nutzung entsprechender Sensoren so kompliziert und ressourcenaufwändig werden, dass dies unterbliebe und individueller wie gesellschaftlicher Nutzen gar nicht erst generiert werden könnte. Es bleibt hier offen, ob eine Regulierung ,,jenseits von Markt und Staat" (Ostrom 1999) oder die Etablierung von Tugenden (Benkler und Nissenbaum 2006) einen Beitrag zur Lösung dieser Herausforderungen leisten können. Denkbar ist jedoch, dass bei Überschreiten einer Obergrenze für die Größe von Gemeinschaften soziale Mechanismen nicht mehr ausreichen, um Missbrauch auszuschließen. 


\section{Validität und Reliabilität der digitalen Allmende}

Ein mit dem bereits Aufgezeigten verbundenes Problem ist spieltheoretischer Natur: Wenn von Citizen Science und Open Science die Rede ist, wird in aller Regel unterstellt, dass sich die jeweiligen AkteurInnen kooperativ verhalten. Diese Annahme muss in der Praxis aber nicht zutreffen, da Szenarien vorstellbar sind, in denen es aus individueller Sicht rational erscheinen mag, zu defektieren, d.h. nicht „mitzuspielen“, und bspw. absichtlich verfälschte Sensordaten zur Verfügung zu stellen. Ein Szenario wäre die Messung von Umweltbelastungen im Umkreis der Lausitzer Braunkohletagebaue - dort stoßen BefürworterInnen und GegnerInnen des Braukohleabbaus auch auf Bürgerebene zu-

\section{Lösungsansätze}

Einen Lösungsansatz, der zuallererst nicht auf technischen Vorkehrungen beruht, sondern auf Vertrauen und Vertrauenswürdigkeit, könnte man als soziale Sensorvalidierung bezeichnen. Für deren Realisierung müssten Organisationsstrukturen entwickelt werden, wie sie sich bspw. in der Open-Source-Szene als digitale Allmende sowie allgemein bei der Verwaltung von Allmendegütern herausgebildet haben. Zentral dafür wäre, dass Signalisierungsmöglichkeiten für Vertrauen und Vertrauenswürdigkeit entwickelt und betrieben werden, was allerdings zusätzlichen Ressourcenaufwand mit sich brächte und daher die Bereitschaft zur Entwicklung, Aufrechterhaltung und aktiven

\section{Es sind Szenarien vorstellbar, in denen es aus individueller Sicht rational erscheinen mag, nicht „mitzuspielen“ und absichtlich verfälschte Sensordaten zur Verfügung zu stellen.}

sammen. Beide Seiten hätten durchaus Anlass, nur bestimmte Daten weiterzugeben oder diese gar, entsprechend ihrer Interessen, gezielt zu produzieren.

Die Möglichkeit der Defektion ist besonders dann gegeben, wenn sich die Personen, die eine digitale Allmende bereitstellen oder auch nutzen, wechselseitig nicht kennen und daher soziale Normen, die einem rein egoistischen Verhalten normalerweise entgegenwirken, nicht greifen. In solchen Fällen sind Vertrauen und Vertrauenswürdigkeit schwierig herzustellen. Dies könnte wiederum dazu führen, dass Daten, die mithilfe von Participatory Sensing oder Wearable Technologies gewonnen wurden, nicht in dem Maße genutzt werden, wie es möglich und vielleicht auch gesellschaftlich wünschenswert wäre. Dann läge eine Unternutzung nicht wegen fehlender durchsetzbarer Regeln, sondern mangels Vertrauen vor. Das meist positiv konnotierte Prinzip der partizipativen und mit geringen Einstiegshürden verbundenen Einbindung vieler individueller und korporativer AkteurInnen wird somit zu einem Risiko, da nicht ohne Weiteres sichergestellt werden kann, dass die Daten tatsächlich valide und reliabel sind. Die Gewährleistung einer validen und reliablen Datenbasis - neben Sensorqualität, Vorkehrungen gegen Fehlbedienung und Ähnlichem - erscheint daher als eine der großen Herausforderungen für den flächendeckenden Einsatz von Sensoren als Werkzeuge von Citizen Science und Open Science, während gleichzeitig eine allgemeine Nutzung offener Datenquellen durchaus Anreize zur absichtlichen Verfälschung mit sich bringt. Wie also gegenüber den Sensordatennutzern Quality of Data (QoD) garantiert werden kann, wird für andere Bereiche des Internet of Things, beispielsweise Smart Cities, bereits intensiv diskutiert. Untersuchungen zur Übertragbarkeit der dort vorgeschlagenen Lösungsansätze auf die speziellen Gegebenheiten von Participatory Sensing und Wearable Technologies erscheinen daher ausgesprochen vielversprechend.
Nutzung einer Infrastruktur für Participatory Sensing und Wearable Technologies mindern könnte. Um den Aufwand der angesprochenen sozialen Signalisierungsmöglichkeiten zu reduzieren, existieren verschiedene Ansätze, welche die Abbildung von Vertrauen und Vertrauenswürdigkeit in technischen Infrastrukturen vorantreiben. Im sogenannten Sozialen Internet der Dinge können Sensoren analog zu menschlichen sozialen Netzwerken autonom verschiedene Beziehungen zueinander eingehen (zum Überblick Atzori et al. 2011; zu Lösungsansätzen Nitti et al. 2014; Nitti et al. 2015). Auf dieser Grundlage lassen sich anschließend Werte für die Vertrauenswürdigkeit einzelner Sensoren berechnen. Diese basieren auf der Beziehung der Sensoren zueinander, auf vergangenen „Erfahrungen“ früherer „Zusammenarbeit" sowie auf den Erfahrungswerten der ,befreundeten“ Sensoren und des Dienstbetreibers (Nitti et al. 2012). Alternative Ansätze, welche ohne Berechnungen innerhalb der Sensoren und Netzwerke auskommen, setzen auf die Beobachtung des Verhaltens einzelner Sensoren und der von ihnen gelieferten Daten (Quan et al. 2012). So lassen sich automatisierte Regressionsanalysen nutzen, um (bewusst) fehlerhafte bzw. nicht plausible Sensordaten erkennen und aus der weiteren Verarbeitung oder Analyse ausschließen zu können. Hierbei stützt man sich vor allem auf das Vorhandensein vergangener Werte eines Sensors oder Werte gleichartiger Sensoren in der näheren Umgebung (Javed und Wolf 2012). Ebenso finden sich in der Literatur Ansätze für monetäre Anreize zur Bereitstellung wahrheitsgemäßer Messwerte, wobei davon ausgegangen wird, dass sich die Korrektheit der gelieferten Werte entweder nachträglich oder abermals anhand benachbarter Sensoren verifizieren lässt (Faltings und Radanovic 2017). Darüber hinaus finden auch in neuartigen Sensornetzwerken etablierte Sicherheitsmechanismen wie sogenannte Trusted Computing-Ansätze oder leichtgewichtige Verschlüsselung sowohl an den Sensoren selbst (Ukil et al. 
2011) als auch in den sie verbindenden Netzwerken (Kothmayr et al. 2013) Anwendung. Diese können aber einer aktiven Verfälschung von Werten durch defektierende SensorbetreiberInnen nicht entgegenwirken.

Um möglichst viele Akteure dazu zu bewegen, Sensoren zu nutzen und Daten bereitzustellen, schlagen Restuccia et al. (2016) vor, Anreize zu bieten und nennen neben (sehr kleinen) Geldbeträgen auch andere Belohnungsmodelle, so beispielsweise exklusiven Zugriff auf die gesammelten Daten oder auf andere, aus Sicht der potenziell Beitragenden, attraktive Ressourcen. Technisch ließen sich solche dem Prinzip der Reziprozität folgende Ansätze vergleichsweise einfach umsetzen, indem z. B. die Auflösung der bereitgestellten Daten (minütliche oder stündliche Werte), die Antwortgeschwindigkeit oder auch die Anzahl maximal möglicher Anfragen abhängig von der Menge (und Qualität) der durch den anfragenden Akteur bereitgestellten Daten ist. Derartige technisch durchgesetzte Reziprozitätsmechanismen sind bereits aus dem Peer-to-Peer-Filesharing der 2000er-Jahre bekannt (Feldman und Chuang 2005) und ließen sich grundsätzlich auf den Bereich der Sensordatenbereitstellung übertragen. Neben der puren Datenmenge ließe sich dabei gegebenenfalls auch die Datenqualität und die durch die jeweils bereitgestellten Daten bediente „Nachfrage“ (z. B. „Wie oft wird ein bereitgestellter Feinstaubwert an einem bestimmten Ort von anderen Akteuren abgefragt" ${ }^{\star \prime}$ berücksichtigen und somit einem ansonsten bestehenden Anreiz, lediglich große Datenmengen minderer Qualität bereitzustellen, entgegenwirken.

Solche Lösungen bringen jedoch selbst Probleme mit sich: So widerspricht ein bevorzugter oder gar exklusiver Zugriff auf die gesammelten Daten der Grundidee von Citizen Science und Open Science. Monetäre Anreize wiederum gehen fast notwendigerweise mit einer Kommerzialisierung einher, die zumindest vielen Auffassungen von Open Science entgegenläuft und zudem oftmals an der Verfügbarkeit entsprechender Gelder scheitern wird. Nicht zuletzt bergen monetäre Anreize das Risiko, andere (insbesondere intrinsische) Motivationsfaktoren abzuschwächen (siehe beispielsweise die Beiträge in Frey und Osterloh 2001).

\section{Fazit}

Wie sich Participatory Sensing und die Nutzung von Wearable Technologies als Werkzeuge von Open Science und Citizen Science in Zukunft entwickeln werden, ist nur schlecht zu prognostizieren, da hier zahlreiche AkteurInnen beteiligt sind, die nicht innerhalb von institutionellen Rahmungen handeln - dies erschwert die Identifikation von Zielen und Motiven deutlich. Außerdem stoßen in diesem Feld einzelne Personen, Nichtregierungsorganisationen wie beispielsweise Bürgerinitiativen, ForProfit- und Non-Profit-Organisationen sowie staatliche Institutionen mit teilweise sehr unterschiedlichen Zielsetzungen und Handlungsmöglichkeiten aufeinander. Der rasante und oftmals nicht vorhersehbare technische Wandel, der in den letzten Jahren beispielsweise zur Miniaturisierung und Verbilligung von Sensoren geführt hat, trägt seinen Teil zur schlechten Prognostizierbarkeit des Feldes bei. Inwieweit Technik in großem Maßstab tatsächlich erfolgreich als Werkzeuge von Citizen Science und Open Science verwendet werden kann, muss daher offenbleiben. Sicherlich lassen sich zahlreiche Szenarien entwickeln, die wünschenswerte und weniger wünschenswerte oder plausible und weniger plausible Zukünfte skizzieren - mehr jedoch nicht. Besonders hinsichtlich der Frage, ob entsprechende Technologien durchsetzbare Regeln benötigen, um Missbrauch zu verhindern, aber gleichzeitig die Vorteile erhalten können, ist dies eine wenig komfortable Situation. Eines scheint jedoch gewiss: Nicht so sehr die Verfügbarkeit von technischen Lösungen zur Unterstützung von Open Science und Citizen Science wird diese disruptiv wirken lassen, sondern (wenn überhaupt) die allgemeine Bereitschaft, diese Technik produktiv zur Lösung der skizzierten Herausforderungen einzusetzen.

\section{Literatur}

Atzori, Luigi; Iera, Antonio; Morabito, Giacomo (2011): SIoT: Giving a Social Structure to the Internet of Things. In: IEEE Communications Letters 15 (11), S. 1193-1195. DOI: 10.1109/LCOMM.2011.090911.111340. Austen, Kat (2015): Pollution Patrol. In: Nature 517 (7533), S. 136-138. DOI: 10.1038/517136a.

Benkler, Yochai; Nissenbaum, Helen (2006): Commons-based Peer Production and Virtue. In: Journal of Political Philosophy 14 (4), S. 394-419. DOI: 10.1111/j.1467-9760.2006.00235.x.

Buchanan, James M.; Yoon, Yong J. (2000): Symmetric Tragedies: Commons and Anticommons. In: Journal of Law and Economics 43 (1), S. 1-14. DOI: $10.1086 / 467445$.

Devarakonda, Srinivas et al. (2013): Real-time Air Quality Monitoring through Mobile Sensing in Metropolitan Areas. In: Proceedings of the $2^{\text {nd }} A C M$ SIGKDD international workshop on urban computing. New York: ACM, S. 1-8. DOI: 10.1145/2505821.2505834.

Faltings, Boi; Radanovic, Goran (2017): Mechanismen zur Beschaffung korrekter Daten. In: Informatik Spektrum 40 (1), S. 64-74. DOI: 10.1007/s00287-016-1010-5.

Fecher, Benedikt; Friesike, Sascha (2014): Open Science: One Term, Five Schools of Thought. In: Bartling, Sönke; Friesike, Sascha (eds.): Opening Science. Cham: Springer, S. 17-48. DOI: 10.1007/978-3-319-00026-8_2.

Feldman, Michal; Chuang, John (2005): Overcoming Free-riding Behavior in Peer-to-peer Systems. In: ACM SIGecom Exchanges 5 (4), S. 41-50. DOI: 10.1145/1120717.1120723.

Frey, Bruno S.; Osterloh, Margit (Hg.) (2001): Successful Management by Motivation: Balancing Intrinsic and Extrinsic Incentives. Berlin: Springer.

GEWISS (2016): Grünbuch Citizen Science Strategie 2020 für Deutschland. Berlin: Projekt „Bürger schaffen Wissen - Wissen schafft Bürger“ (GEWISS). Online verfügbar unter https://www.leibniz-gemeinschaft.de/fileadmin/ user_upload/downloads/Forschung/Citizen_Science/Gruenbuch_Citizen_ Science.pdf, zuletzt geprüft am 12.06.2017.

Goldman, Jeffrey et al. (2009): Participatory Sensing: A Citizen-powered Approach to Illuminating the Patterns that Shape our World. Foresight and Governance Project White Paper. Center for Embedded Networked Sensing. Online ver- 
fügbar unter https://www.wilsoncenter.org/sites/default/files/participatory_ sensing.pdf, zuletzt geprüft am 12.06.2017.

Hardin, Garrett (1968): The Tragedy of the Commons. In: Science 162 (3859),

S. 1243-1248. DOI: 10.1126/science.162.3859.1243.

Javed, Nauman; Wolf, Tilman (2012): Automated Sensor Verification using Outlier Detection in the Internet of Things. In: $32^{\text {nd }}$ International Conference on Distributed Computing Systems Workshops. IEEE, S. 291-296. DOI: 10.1109/ICDCSW.2012.78.

Kothmayr, Thomas; Schmitt, Corinna; Hu, Wen; Brünig, Michael; Carle, Georg (2013): DTLS Based Security and Two-way Authentication for the Internet of Things. In: Ad Hoc Networks 11 (8), S. 2710-2723.

DOI: 10.1016/j.adhoc.2013.05.003.

Kumar, Prashant et al. (2015): The Rise of Low-cost Sensing for Managing Air Pollution in Cities. In: Environment International 75, S. 199-205. DOI: 10.1016/j.envint.2014.11.019.

Lindeman, Neil (2012): Subjectivized Knowledge and Grassroots Advocacy. In: Journal of Business and Technical Communication 27 (1), S. 62-90. DOI: $10.1177 / 1050651912448871$.

NABU (2016): Stunde der Wintervögel mit Rekordteilnahme. Online verfügbar unter https://www.nabu.de/tiere-und-pflanzen/aktionen-und-projekte/ stunde-der-wintervoegel, zuletzt geprüft am 12.06.2017.

Nitti, Michele; Atzori, Luigi; Cvijikj, Irene P. (2015): Friendship Selection in the Social Internet of Things: Challenges and Possible Strategies. In: IEEE Internet of Things Journal 2 (3), S. 240-247. DOI: 10.1109/JIOT.2014.2384734.

Nitti, Michele; Girau, Roberto; Atzori, Luigi (2014): Trustworthiness Management in the Social Internet of Things. In: IEEE Transactions on Knowledge and Data Engineering 26 (5), S. 1253-1266. DOI: 10.1109/TKDE.2013.105.

Nitti, Michele; Girau, Roberto; Atzori, Luigi; Iera, Antonio; Morabito, Giacomo (2012): A Subjective Model for Trustworthiness Evaluation in the Social Internet of Things. In: IEEE 23rd International Symposium on Personal Indoor and Mobile Radio Communications (PIMRC). IEEE, S. 18-23. DOI: 10.1109/ PIMRC.2012.6362662.

Olson, Mancur (1965): The Logic of Collective Action: Public Goods and the Theory of Groups. Cambridge M. A.: Harvard University Press.

Ostrom, Elinor (1999): Die Verfassung der Allmende: Jenseits von Staat und Markt. Tübingen: Mohr Siebeck.

Pacheco-Torgal, Fernando et al. (2016): Start-up Creation: The Smart Eco-efficient Built Environment. Duxford: Woodhead.

Quan, Zhou; Gui, Fu; Xiao, Deqin; Tang, Yi (2012): Trusted Architecture for Farmland Wireless Sensor Networks. In: IEEE $4^{\text {th }}$ International Conference on Cloud Computing Technology and Science (CloudCom). IEEE, S. 782-787.

Restuccia, Francesco; Das, Sajal K.; Payton, Jamie (2016): Incentive Mechanisms for Participatory Sensing: Survey and Research Challenges. In: ACM Transactions on Sensor Networks 12 (2), 13, 40 S. DOI: 10.1145/2888398.

Ruiz-Mallén, Isabel et al. (2016): Citizen Science. In: Science Communication 38 (4), S. 523-534. DOI: 10.1177/1075547016642241.

Scott, Dayna Nadine (2015): „We Are the Monitors Now.. In: Social and Legal Studies 25 (3), S. 261-287. DOI: 10.1177/0964663915601166.

Swan, Melanie (2012): Sensor Mania! The Internet of Things, Wearable Computing, Objective Metrics, and the Quantified Self 2.0. In: Journal of Sensor and Actuator Networks 1 (3), S. 217-253. DOI: 10.3390/jsan1030217.

Ukil, Arijit; Sen, Jaydip; Koilakonda, Sripad (2011): Embedded Security for Internet of Things. In: $2^{\text {nd }}$ National Conference on Emerging Trends and Applications in Computer Science (NCETACS). IEEE, S. 1-6. DOI: 10.1109/ NCETACS.2011.5751382.
Vayena, Effy; Tasioulas, John (2015): „We the Scientists“: a Human Right to Citizen Science. In: Philosophy and Technology 28 (3), S. 479-485. DOI: 10.1007/ s13347-015-0204-0.

Vaz, Paulo; Bruno, Fernanda (2003): Types of Self-surveillance: From Abnormality to Individuals 'at Risk'. In: Surveillance and Society 1 (3), S. 272-291.

Whitson, Jennifer R. (2013): Gaming the Quantified Self. In: Surveillance and Society $11(1 / 2)$, S. 163-176.

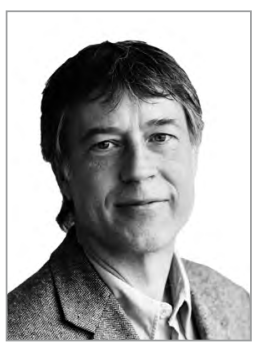

\section{PROF. DR. KARSTEN WEBER}

ist Ko-Leiter des Instituts für Sozialforschung und Technikfolgenabschätzung (IST) der OTH Regensburg. Er beschäftigt sich derzeit mit individuellen und gesellschaftlichen Auswirkungen von luK-Technologie sowie mit wertebasierter Gestaltung von Technik insbesondere im Gesundheitsbereich.

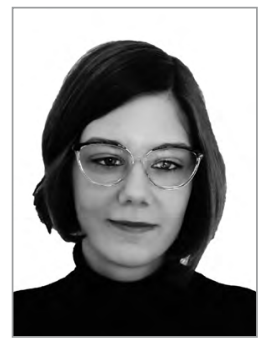

\section{NADINE KLEINE}

ist Forschungsassistentin im Forschungscluster „Ethik, Technologiefolgenforschung und Nachhaltige Unternehmensführung" (ETN) und wissenschaftliche Mitarbeiterin am IST der OTH Regensburg. Sie beschäftigt sich insbesondere mit techniksoziologischen Fragestellungen zu gesellschaftlichen Auswirkungen von innovativen Technologien. Ihr aktueller Forschungsgegenstand sind Wearable Technologies.

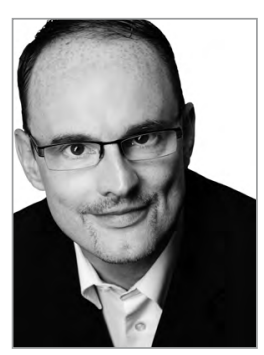

\section{DR.-ING. FRANK PALLAS}

ist Senior Researcher am Fachgebiet Information Systems Engineering der TU Berlin. Neben Fragen des Privacy Engineering befasst er sich auch mit institutionenökonomischen und governancebezogenen Aspekten moderner luK-Technologie - insbesondere Cloud/Fog Computing und IOT sowie den daraus erwachsenden Implikationen für die konkrete Technologiegestaltung.

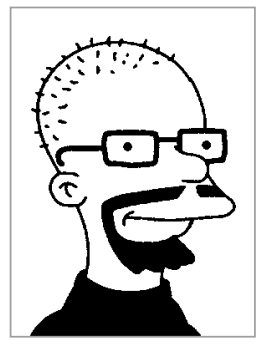

\section{MAX-R. ULBRICHT}

ist Diplominformatiker und arbeitet als wissenschaftlicher Mitarbeiter am Fachgebiet „Information Systems Engineerung" der TU Berlin. Neben Fragen der Regulierung von und durch Technologie liegen seine Forschungsschwerpunkte in den Bereichen Privacy Enhancing Technologies, Consent Management sowie Privacy-Preserving Data Integration. 